\title{
A brief review of mathematical models concerning the vertical variation of the water density in the sea
}

\author{
R. Purisi $\left(^{*}\right)-$ A. Rovellit $(* *)-$ E. Silusti $(* *)$ \\ Received on October 10th, 1976
}

Sumary. - This paper is concerned with a fundamental marine problem, i.e. the internal waves. After a discussion of the physical assump. tions about some mathematical models describing the vertical structure of the sen, we present the various driving fores and the different theoretieal formalisms to approach the problem.

Russunto. - Nello stulio dei fenomeni comnessi alla dinamica marina, il problema delle onde inteme riveste un ruolo di primaria inportanza. In quosto lavoro si analizzano inizialmente alemi modelli che rignardano la formazione di determinate struture verticali del mare, indieando le limitazioni che comportano aleme ipotesi ivi utilizzate. Introdotta poi l'equazione che deserive le onde interne e dopo averne costatata la similariti con lequazione di sehroedinger, si passa ad una rassegna dei differenti approcei matematici che avviano a soluzione tale problema.

\section{1. - INtroductiox}

The purpose of this note is to introduce a marine problem, that of the internal waves. These are related to the vertical variation of the water density, called "thermocline". These waves are describer by a Schroedinger-type equation; also the Lagrangian formalism and some techniques of the quantum field theory have been used in this

(*) Istituto di Fisica dell'Atmosfera (CNR) P.le Sturzo, 31 - Roma.

(**) Istituto Nazionale di Geofisiea - Roma.

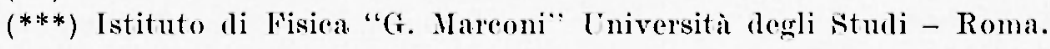


kind of problems. Because these formalisms have been widely used by experts in quantum theory, we feel that an exchange of informations could be fruitful and, in this case, also possible.

To describe the phenomena, we start by remembering that the experimental temperature and salinity of the sea water have a rariation - sometimes a sharp one - about $10 \div 50$ meters under the sea level. This variation is called "thermocline" and it implies a $2 \%$ variation of the sea water density. The main reason of this variation is due to the exchange of the heat and of turbulence with the atmosphere. Other reasons are related to the Coriolis force and to the real shape of the oceanic basins. We will describe first how deep is our knowlerge of the effect of these parameters on the depth "d" of the thermocline (\$ 2$)$. The presence of this variation of the density " $Q$ " of the sea water allows the existence of some waves, called "internal waves". To have an intuitive picture one can think to the surface waves (considered as waves of the discontinuity surface between air and water), remembering that the internal waves are larger and slower. Their characteristic times can be $10 \div 30$ minutes and some amplitules of about $20 \div 30$ meters have been observer. By a theoretical point of view, if one linearizes the Fuler equation and uses the Boussinesq approximation (it will be rescribed in $\$ 4$ ), one arrives to a Schroedinger-type equation. These well known results allows us a reasonable insight of the problem but suggest us various questions.

There are many sources of internal waves; so there is the probability that in every point of the sea one can see at one time more than one internal waves. This happens in practice, Garrett and Munk $\left(^{6.7}\right)$ gived an empirical formula to know the local density of probability of internal waves. A more open question concerns indeed the energetic balance of these internal waves. Their dissipation by friction being rather small, the reason of their dlecay is not very clear. $\Lambda$ bont their origin we have a rather large number of sources: storms, interaction of currents with bottom irregularities, tides, non-linear exchanges between surface and internal waves. These are discussed in last section.

\section{THE THERHOCLINE}

Let us consider now the air-water system. By taking into account only the Coriolis force, the gravity $\mathrm{g}$ and the exchange of heat $Q$ between air and ocean, we want to study under what conditions 
we can arrive to a realistic thermocline. The Euler equations, the continuity relation for the mass and the heat give:

$$
\begin{aligned}
& \frac{\mathrm{d} \mathbf{v}}{\mathrm{d} t}+\Omega \times \mathbf{v}+\frac{1}{\varrho} \nabla p-\mathbf{g}=\frac{1}{\varrho} \mathrm{F} \\
& \frac{\mathrm{d} \omega}{\mathrm{d} t}+\varrho \nabla \cdot \mathbf{v}=0 \\
& \frac{\partial T}{\partial t}=\nabla \cdot(k \nabla T)+\frac{1}{\varrho c} \dot{Q} \\
& \varrho=\varrho(p \cdot I)
\end{aligned}
$$

where $\mathrm{v}$ is the velocity, $p$ the pressure, $\varrho$ the density, $T$ the temperature, $c$ the specific heat, and $\frac{\mathrm{d}}{\mathrm{d} t}=\frac{\partial}{\partial t}+\mathbf{v} \cdot \nabla$ In the preceeding formulae, we assumed that the $x$ axis is directerl to the north, the $z$ axis is directed upward and the $y$ axis is directed eastward. The Coriolis force is described by $f=2 \Omega \sin \varphi$. In the $\beta$-plane approximation one puts

$$
f=f_{0}+\beta\left(y-y_{0}\right)
$$

The difiusion of turbulence in the horizontal and vertical directions is described by the $K_{h}$ and $K_{v}$ coefficients. These equations should be supplemented by their boundary conditions. We can note however that this is a system of non-linear equations of difficult solution. To be able to obtain simpler relations, necessary in practice, one usually introduces some "approximations" (steady conditions, linearization of the velocities etc.) which allow semplified equations

$$
\begin{aligned}
& -f v=-\frac{1}{\varrho} \frac{\partial p}{\varrho x} \\
& f u=-\frac{1}{\varrho} \frac{\partial p}{\partial y} \\
& o=-\frac{1}{\varrho} \frac{\partial p}{\partial z}-g \\
& u \frac{\partial T}{\partial x}+v-\frac{\partial T}{\partial !}+v \frac{\partial T}{\partial z}=K_{h} \Delta_{h} T+K_{v} \frac{\partial^{2} T}{\partial z^{2}} \\
& \nabla . \mathbf{v}=0
\end{aligned}
$$

One is obliged to remark that these equations are not obtained only on physical ground but also because they can have simple solutions of elementary type. 
It is important to note that the temperature $T$ can describe the variations of the density if one assumes that the salinity is rather constant with the depth. Such assumption being acceptable, one can assume

$$
\varrho=\varrho_{o}\left[1-\alpha\left(T-T_{o}\right)\right]
$$

where $\alpha$ is a constant. If one adds this last relation to the [थ] equations, one then arrives to six equations for the unknown quantities: $\varrho, T, p, u, v, u$. The boundary conclitions are now of crucial importance for the various models that will be discussed in the following, which have been mainly obtained after 1960.

A very interesting model is due to Robinson and Stommel ( $\left.{ }^{23}\right)$ where the thermic structure of an ocean has been studied under the hypothesis of geostrophic balance of forces and a north-south coast at $x=0$.

The surface temperature is given as a boundary condition

$$
\begin{array}{ll}
T=T(y)=T_{o}+T_{1} y & \text { at } z=0 \\
T \rightarrow 0 & \text { at } x=0 ; z \rightarrow-\infty
\end{array}
$$

The other boundary condition concerns the vertical velocity

$$
\begin{array}{ll}
w=0 & \text { at } z=0 \\
w \rightarrow w \infty(x, y) & \text { at } z \rightarrow-\infty
\end{array}
$$

Assuming also that one can forget the horizontal turbulent diffusion $\left(K_{h}=0\right)$ and the advenction in the $x$ direction, one can solve the problem with the method of separation of variables:

$$
\begin{aligned}
& T=G(x) \vartheta(\xi) \\
& w=H(x) x(\xi)
\end{aligned}
$$$$
\text { with } \xi=z F(x) \text {. }
$$

It is not obvious naturally that such a position contains all the physically interesting informations. In any case, after some manipulations, one can obtain by transformation of similarity the temperature structure.

One has

$$
T=\eta_{o}(y) e^{-\frac{1}{3 z}\left\lceil\frac{1}{2 K_{h}} \frac{\alpha \beta y}{f^{2}(y)}\left(T_{0}+\frac{T_{1}}{\beta} \rho_{0} 10^{-8}\right)-\frac{1}{x}\right]}
$$


The theoretical behaviour of the temperature $T$ that we have discussed before gives a rather good agreement with some experimental clata (Fig. 1).

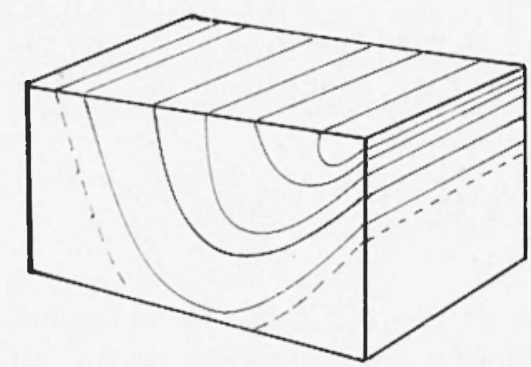

a) theoreticalcalculation

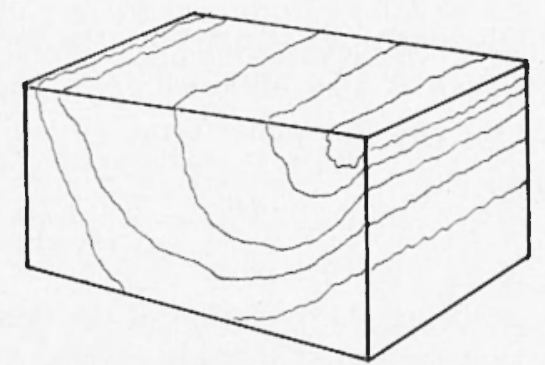

b) experimental data

Fig. 1

$\Lambda$ different approach has been choosen by Velander ${ }^{20}$ ) which remarked the important role that the diffusion of the mass can have. This model is peculiar because the advection is forgotten and the diffusion coefficient is the main physical quantity.

More in particular, Welander assumes an hydrostatic and geostrophic balance of forces. He studies the effect of a variation of the density $\varrho^{\prime}=\varrho-\bar{o}$. The density of the surface and bottom is given as boundary condition:

$$
\begin{array}{ll}
\varrho=\varrho_{o}(\vartheta, \varphi) & \text { at } z=0 \\
\varrho=\text { const } ; \varrho \mathbf{v}=0 & \text { at } z \rightarrow-\infty
\end{array}
$$

The system of equations [2] is then reduced to the equation $\left({ }^{30}\right)$

$$
\partial_{q z} M \partial \vartheta_{z} M-\partial \vartheta z z M \partial_{q z} M=\operatorname{cotg} \vartheta \partial_{\varphi} M \partial_{z z z} M
$$

for the integrated quantity

$$
M(\vartheta, \varphi, z)=\left.\int_{-\infty}^{\tilde{a}} \mathrm{~d} z^{\prime}\right|_{-\infty} ^{z^{\prime}} \mathrm{d} z^{\prime \prime} \varrho(\vartheta, \varphi, z)
$$

Particular solutions can be obtained by introducing a new rariable

and then by putting

$$
\eta=\log \sin \theta
$$

$$
M=P(q, \eta) Q(\eta, z)
$$


One then obtains the solution and it results $\left({ }^{30}\right)$

$$
M=M_{o}(\varphi, \theta) e^{\frac{\hbar z}{\sin \theta}}
$$

Obviously $M_{0}$ is fixed by the boundary condition at $z=0$; the quantity $k$ is also obtained from the boundary conditions. The density also has the same form

$$
\varrho^{\prime} \circ(\theta, \varphi) e^{\frac{K z}{\sin \theta}}
$$

$Q^{\prime}$ o being the variation of the density at $z=0$. It has to be remarked that the value of $k$ is such that at about 1.000 meters the perturbation vanishes. This is in some sense due to the phenomenological data and is not the consequence of the equations. More interesting appears the "sin $\varphi$ " behaviour of the depth of penetration between the equator and the poles. A more realistic approach at the same problem has been introduced by Barcilon (1) by considering the boundary of the ocean both at east and west $(x=0$ and $x=1)$. In formulae, his boundary conditions are:

$$
\begin{array}{ll}
T=0 & \\
u=0 & \text { at } x=0,1 \\
T=0 & \text { at } z=0 \\
w=0 & \\
T=\vartheta(y) & \text { at } z=1
\end{array}
$$

where $\vartheta(y)$ is the surface temperature. Obviously the physically interesting quantities have been normalized by scale quantities, in order to have simpler adimensional quantities

$$
\begin{aligned}
& 0 \leqslant \tilde{x} \leqslant 1 \\
& 0 \leqslant \tilde{y} \leqslant 1 \\
& 0 \leqslant \tilde{T} \leqslant 1
\end{aligned}
$$

Moreover the vertical gradient of the temperature has been approximated by $\nabla T \sim \Delta T / D$ to aroid non-linear terms of this equation. Then 
the use of Fourier transform allows to find the solution. It lats to be remarkerl that this author introduces the perturbation

$$
\tilde{T}=\bar{T}-T
$$

and assumes $\tilde{T} \neq 0$ for $x \rightarrow 0$. It is in this way assumed that a boundary layer is only in the west coast. The explicit form of the temperature is rather complex:

$$
\bar{T}=\left.\frac{2 \vartheta(y)}{\pi}\right|_{0} ^{\infty} \sin (\chi \eta) \frac{1-e^{-\not \eta^{2}}}{\eta} \mathrm{l} \eta
$$

where $\chi$ is

$$
\chi=\left[\frac{(1-z)^{4} S \beta f_{o} L}{(1-x) K_{v}\left(L+\beta f_{o} y\right)^{2}}\right]^{1 / 3}
$$

For the quantity $\tilde{T}$ one has

$$
\tilde{T}=-\frac{2 \vartheta(y)}{\pi} \int_{0}^{\infty} \frac{1-e^{-\eta \eta^{5}}}{\eta} \sin (\eta \zeta) e^{-\xi / \eta^{2}} \mathrm{~d} \eta
$$

where

$$
\begin{gathered}
\zeta=(1-z)\left[\frac{K_{v}}{f_{o} \beta L}\left(L+S \beta f_{o} y\right)^{2}\right]^{-1 / 4} \\
\xi-\frac{x \gamma^{\prime} S \beta f_{o} K_{v}}{K_{h}\left(f_{o}+\beta L y\right)}
\end{gathered}
$$

Then one can calculate analytically $\left(^{1}\right)$

$$
T=\frac{2 \vartheta(y)}{1 \sqrt{6 \pi x}} \cos \left(\frac{3 \sqrt{3} \chi}{4^{1 / 3} 8}+\frac{\pi}{6}\right) e^{-\frac{3}{4^{1 / 3}}}
$$

From a graphical representation of the isothermes in the $(x, z)$ plane, we can see easily an "asymmetry" between eastern and western coasts; in particular a boundary layer exists solely along the Western coasts.

One can now remark in general that the preceeding morlels have some features in common. They want to treat analitically a rather complicated problem and it is obvious that some general aspects of the physical situation must be lost. In particular one must stress that to disregard the arlvective parts of $\frac{d v}{a c}$ or $\frac{d a}{a c}$ implies that some 
important informations about the interchange of kinetic and potential energies are lost. More in particular, if one assumes a static balance of forces as the hychostatic and the geostrophic force, then one obtain essentially solutions of exponential type. All the variations are related with the initial or boundary conditions. It is obvious that in this way one can obtain only a rough picture which is also more unsatisfactory if one thinks that the realistic thermocline is not only a rather complex profile, but also it has many horizontal discontinuity surfaces followed by small well mixed layer (the finestructure of thermocline) ( $\left.{ }^{31}\right)$. One has then to take into account other aspects as the surface input of turbulance and the flow between kinetic, thermic and potential energies. Turner and Krauss $(12,13)$ have stressed the importance of these aspects. They remarked: "most of these preceding morlels and other studies not citer suffer from two disarantages. They often contain more than one physical process in a way that makes it difficult to disentangle them and they are nearly all stearly state calculations".

In (12), a work of main phenomenological and experimental character, the seasonal variation of thermocline is studied. It results experimentally that in winter one can see a very depth and small thermocline while in summer one has in the density sharp discontinuity. This is explained by taking into account the potential energy $E$ of a two-fluids system (the first of density $\varrho$ and depth $h$, the second of larger density $\varrho+\Delta \varrho$ and depth $d-h$ ). One has

$$
E=g h \Delta \varrho\left(\frac{h}{2}-d\right)
$$

Remarking now that one can say that

$$
\text { gh } \Delta \varrho=\text { const. }=b
$$

where $b$ is the total buoyancy. One has, for the rate of change of potential energy $E\left({ }^{12}\right)$

$$
\frac{\mathrm{d} E}{\mathrm{~d} t}=\frac{1}{2}(g h \Delta \varrho) \frac{\mathrm{d} h}{\mathrm{~d} t}=\frac{1}{2} b \frac{\mathrm{d} h}{\mathrm{~d} t}
$$

In this case, fixing the rate of input of mechanical energy and no buoyancy flux across the sea surface, the well-mixed layer will discend at a constant rate, which is inversely proportional to the total buoyancy $b$. 
In the second work the theoretical aspects are studied in more detail, the horizontal low of energy is studied

$$
\frac{\partial T}{\partial t}+\frac{\partial}{\partial z}\left(w^{\prime} T^{\prime}\right)=\dot{Q}
$$

where $T^{\prime}=T-T$ and $w^{\prime}$ is the perturbation of the vertical velocity. The buoyancy term $\overline{w^{\prime}} T^{\prime}$ is linked to the level $h$ of the thermocline by the empiric position ( $\left.{ }^{13}\right)$.

$$
\left.\overline{\left(w^{\prime} T^{\prime \prime}\right.}\right)_{z+h}=\left[\left(T_{0}-T_{h}\right) \frac{\mathrm{d} h}{\mathrm{~d} t}\right] \Lambda\left(\frac{\mathrm{d} h}{\mathrm{~d} t}\right)
$$

where $T_{h}$ is the temperature of the layer directly under $h$ and

$$
\Lambda(x) \begin{cases}0 & x<0 \\ 1 & x \geqslant 0\end{cases}
$$

This implies that if the buoyancy term arrives at $h$, then $h$ goes down, in the other case it remains at the preceding position. From these equations a very complex non-linear equation is obtained. This is however solved with some approximations and one obtains (13)

$$
\begin{gathered}
\frac{\mathrm{d} T_{s}}{\mathrm{~d} t}=\frac{2}{h^{2}}[(S+B) h-((+-D+S / \beta)] \\
\Lambda\left(\frac{\mathrm{d} h}{\mathrm{~d} t}\right) \frac{\mathrm{d} h}{\mathrm{~d} t}=\frac{1}{\left(T_{s}-T_{h}\right) h_{h}} 2[((t-D+S / \beta)(S+B) h]
\end{gathered}
$$

where $G$ is the kinetic energy, $S$ the value of the solar radiation adsorbed in the sea, $\beta$ its attenuation factor and $B$ the sum of the terms which concern the thermic exchanges at the surface. It is to be remarerl the good agreement with the experimental data; some discrepances being perinaps due to dissipative or arlvective effects forgotten in the computations.

I more sophisticaterl version is rue to Denmann $\left({ }^{3}\right)$.

\section{The Fine-struCture of the therMocine}

The results of the precerling sections can be userl to study the fine-structure of thermocline. It has been observed [Woods $\left.\left({ }^{31}\right)\right]$ that in strongly stratified regions one can see horizontally some stronger 
cliscontinuities followed by layers of constant temperature. Now, although the horizontal rlimension of the discontinuities are of some centimeters and of the homogeneous layers of few meters, the horizontal dimensions can be of some miles.

It has also to be remarked that the motion in the layers can be a three dimensional turbulence, while in the sheets the strong stratification allows a laminar motion only. At the light of the precerling morlels one can try to interpret this structure as a "memory" of preceding periods of stronger exchange of turbulence between air and water. In these periods a remarkable quantity of turbulence flows from air to water; then this turbulence goes deejer and enlarges $h$, as Turner and Krauss describer. After one other period of strong turbulent exchange, one has a layer under the new thermocline as result of the situation. In any case, some researches have analized the consequences of this horizontal layers whitout speculating about its origin. Submerging a sensor in one point of the oceanic thermocline, the observed spectra of waves are mainly determined by the internal motions, that is, waves between the two neighbour layers which will be rescriber in next paragraph.

More in particular, when an intemal wave gives periodic oscillations to the system, the measured quantities have a well defined motion. Assuming that the temperature gradient is continuous and slowly varying, the rertical oscillation 5 of a fluid element is relater to a variation of the temperature $\theta$ by an approximated linear relation

$$
\zeta=\left(\frac{\overline{\Delta \vartheta}}{\Delta z}\right)^{-1} \vartheta
$$

In presence of a strong stratification this relation cannot be userl. In an interesting work Phillips (ํ) stuclies the spectrum of density fluctuations for a sheet of pure discontinuity near a laver of a constant temperature. Calling $g_{o}(\approx)$ the undisturber density of the fluirl, for a oscillation $\zeta(t)$ of an isopienic surface at the equilibrium, the new rlensity is

$$
\varrho(t)=\varrho_{o}[z-\zeta(t)]
$$

Being all these phenomena of periorlic type with period $T$, we can expand in Fourier series

$$
Q(t)=\sum_{-\infty}^{+\infty} e_{n} e^{i m w_{0} l} \quad \text { with } \omega_{1}=\frac{2 \pi}{T}
$$


The $c_{n}$ coefficients are

$$
c_{n}=\frac{w}{2 \pi} \int_{-T / 2}^{+T / 2} \varrho(t) \mathrm{e}^{-i n w_{n} t} \mathrm{~d} t=-\left.\frac{i}{2 \pi n}\right|_{-T / 2} ^{+T / 2} \frac{\partial o}{\partial t} e^{-i n \omega_{0} t} \mathrm{~d} t
$$

The structure of the density that has been describer implies

$$
\frac{\partial \varrho}{\partial t}=\Sigma_{r}(\Delta \varrho)_{r} \delta\left(t-t_{r}\right)
$$

if one assumes that the "sensor" crosses the sheet of discontinuity $(\Delta o)_{r}$ at the time $t_{r}$.

Then one has

$$
c_{n} c_{n}^{*}=\frac{1}{4 \pi n^{2}} \sum_{r, s}(\Delta \varrho)_{r}(\Delta \varrho)_{s} e^{-i n \omega_{o}\left(t_{r}-t_{s}\right)}
$$

Assuming now that the characteristic time

$$
\tau_{r s}=t_{r}-t_{s}
$$

is not related to the corresponding amplitude and that it is rather larger than $T$,

$$
n \tau_{r, s} \gg T
$$

one has

$$
\overline{c c_{n}^{*}} \simeq \frac{1}{4 \pi^{2} n^{2}} \sum_{r} \overline{(\Delta \varrho)_{r}^{2}}=\frac{{ }^{n} T}{4 \pi^{2} n^{2}} \overline{(\Delta \varrho)^{2}}
$$

where $v$ is the average number of crossing for unit time. Then one can say that the variance of the coefficients of the Fourier transform goes asymptotically as $n^{-2}$. A more general result can be obtained if one assumes

$$
\frac{\partial ?}{\partial t}=\sum r(\Delta Q)_{r} f\left(t-t_{r}\right)
$$

where $f$ is a smoother relta function.

\section{IYTERYAL WAVES}

Let us now describe the periodic motion of the above described horizontal layers. Let us consider the theory of a fluid of density $\varrho$, local velocity $\mathrm{v}=(u, x, w)$ and pressure $\tilde{p}(x, y, z, t)$. Calling g the 
gravity, 9 the Coriolis parameter and $F$ the other external forces, the equation of motion are

$$
\varrho \frac{\mathrm{d} \mathbf{v}}{\mathrm{d} t}+\varrho \mathbf{\varrho} \times \mathbf{v}+\nabla \tilde{p}-\varrho \mathbf{g}=\mathbf{F}
$$

The equation of continuity and the state equation give

$$
\frac{\mathrm{d} \varrho}{\mathrm{d} t}+\varrho \text { div } \mathbf{v}=0 ; \quad \varrho=\varrho(p, T)
$$

One can express $\frac{d}{a l}$ as

$$
\frac{\mathrm{d}}{\mathrm{d} t}=\frac{\partial}{\partial t}+\mathbf{v} \cdot \nabla
$$

In order to obtain some treatable relations one has to introrluce some approximations. The vertical motions being small one can say

$$
\frac{\partial p^{(0)}}{\partial z}=-g \varrho^{(0)}(z)
$$

at first order.

If one now assumes that the fluid is incompressible from the continuity equation one has

$$
\frac{\left(\lg ^{(0)}\right.}{\mathrm{d} t}-0=\operatorname{liv} \mathbf{v}=\frac{\partial u}{\partial x}+\frac{\partial v}{\partial y}+\frac{\partial w}{\partial z}
$$

In this context one is leal to introduce two small quantities

$$
p=\tilde{p}-p^{(0)} ; \quad \delta=\underline{\varrho}(x, y, z, t)-\underline{Q}^{(0)}(z)
$$

The equations of motion now give

$$
\frac{\mathrm{d} \mathbf{v}}{\mathrm{di}}+\boldsymbol{Q} \times \mathbf{v}+\frac{1}{\varrho}-\nabla p-\frac{\delta}{\varrho} \mathbf{g}=\mathbf{F}
$$

and from the continuity equation one has

$$
\frac{\mathrm{d} \varrho}{\mathrm{i} t}=0=\frac{\partial}{\partial t} \delta+u \frac{\partial}{\partial x} \delta+n \frac{\partial}{\partial y} \delta+w \frac{\partial}{\partial z} \delta
$$

the difference between

$\frac{1}{\varrho} \nabla p$ and $\frac{1}{\varrho^{(0)}} \nabla p$ and between $\frac{\delta}{\varrho^{(0)}}$ and $\frac{\delta}{\varrho}$ being smaller, we obtain the results of the classical Boussinesq approximation 


$$
\begin{gathered}
\frac{\mathrm{l} \mathbf{v}}{\mathrm{d} t}+\mathbf{Q} \times \mathbf{v}+\frac{1}{\rho^{(0)}} \nabla p-b \frac{\mathbf{g}}{\mathbf{g}}=\mathbf{F} \\
\nabla \cdot \mathbf{v}=0 \\
\frac{\mathrm{d} \omega}{\mathrm{d} t}=\frac{\partial \delta}{\partial t}+u \frac{\partial \delta}{\partial x}+v \frac{\partial \delta}{\partial !}+w \frac{\partial \rho^{(0)}}{\partial z}=\mathbf{0}
\end{gathered}
$$

The quantity $b$ is callerl specific buoyancy. Approximatly one has $\left({ }^{20}\right)$

$$
\frac{\mathrm{d} b}{\mathrm{~d} t}=-N^{2} x ; \quad N^{2}=-g \frac{\mathrm{d}}{\mathrm{d} z} \log \varrho^{(0)}
$$

where $N$ is the Brunt-Väisäla frequency. It has a central importance in the analysis of these problems; its physical meaning is that of the natural frequency of horizontal oscillations for a vertical column of water around the equilibrium. Now one can forget the Coriolis forces (being the dimensions of these waves rather small) and the non-linear terms of the equations of motion. This is due to the rather small velocities considered. Then one arrives to $\left.{ }^{(20}\right)$

$$
\begin{gathered}
\frac{\partial u}{\partial t}+u \frac{\partial u}{\partial x}+v \frac{\partial u}{\partial y}+w \frac{\partial u}{\partial z}+\frac{1}{Q^{(o)}}-\frac{\partial p}{\partial x}=F_{1} \\
\frac{\partial v}{\partial t}+u \frac{\partial v}{\partial t}+v \frac{\partial v}{\partial t}+w \frac{\partial v}{\partial t}+\frac{1}{Q^{(o)}} \frac{\partial p}{\partial y}=F_{2} \\
\frac{\partial w}{\partial t}+u \frac{\partial w}{\partial x}+v \frac{\partial w}{\partial y}+w \frac{\partial w}{\partial z}+\frac{1}{\rho^{(o)}} \frac{\partial p}{\partial z}-b=F \\
\frac{\partial u}{\partial x}+\frac{\partial v}{\partial y}+\frac{\partial w}{\partial z}=0 \\
\frac{\partial \delta}{\partial t}+w \frac{\partial o^{(o)}}{\partial z}=0
\end{gathered}
$$

With some cross differentiation one then arrives $\left({ }^{22}\right)$ to

$$
\left|\frac{\partial^{2}}{\partial t^{2}}\left(\frac{\partial^{2}}{\partial x^{2}}+\frac{\partial^{2}}{\partial y^{2}}+\frac{\partial^{2}}{\partial z^{2}}\right)+N^{2}(z)\left(\frac{\partial^{2}}{\partial x^{2}}+\frac{\partial^{2}}{\partial y^{2}}\right)\right| w=0
$$

This equation is still rather complicated. One then assumes to sturly waves of the type

$$
w(x, y, z, t)=\mathbb{W}(z) e^{i(k x-n t)}
$$

which are the most common in the experimental situations. Then one has

$$
\frac{\mathrm{d}^{2}}{\mathrm{~d} z^{2}} W(z)+\frac{N^{*}(z)}{i^{\prime}} \cdot l^{2} W(z)=l^{2} W(z)
$$


The boundary conditions of the equation are

$$
\boldsymbol{\sigma} \cdot \mathbf{n}=\mathbf{0} \quad \mathbf{n} \text { is now the normal to the bottom }
$$

For flat bottom at $z=-d$ this is

$$
\text { W }(z=-\mathrm{d})=0
$$

At the free surface $z=\zeta(x, y, t)$ one has

$$
w(z=\zeta)=\frac{d_{\zeta}^{\circ}}{\mathrm{d} t}
$$

With the preceding approximation this furnishes

$$
\frac{\partial^{2}}{\partial t^{2}} w+g \frac{\partial}{\partial z} w=\left.0\right|_{z=\tau(x, y, l)}
$$

and this gives for $W(z)\left({ }^{(21}\right)$

$$
g \frac{\mathrm{d}}{\mathrm{d} z} \mathrm{~W}=n^{2} W
$$

If moreover we assume a rigirl upper surface, then one has also

$$
W(0)=0
$$

These positions give an equations very similar to the well known Schroedinger equation. Our intermal waves correspond formally to the quantum mechanical motion of a particle on a segment. One must however remark that this equation is not obtained in the most clear way. The approximations will be discussed in the following part of this work. In any case, this classical formulation is useful at least to introduce a fruitful language.

We will now rlescribe the most simple case of solutions. It is, from the beginning, clear that where $n^{2}>N^{2}$ one has an exponential behaviour and where $n^{2}<N^{2}$ one as oscillations on $z$ (Fig. 2).

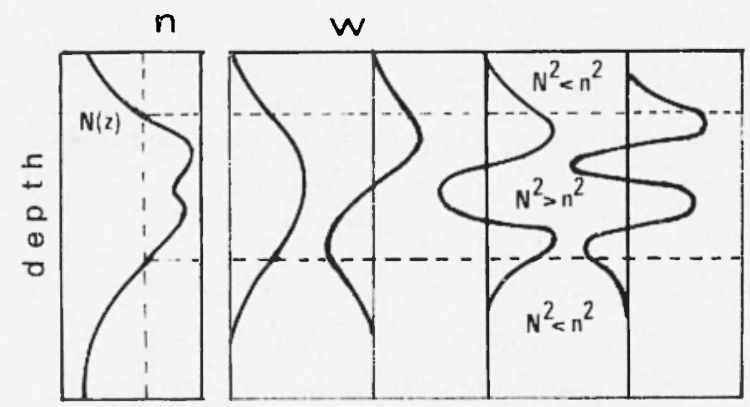

Fig. 2 
Assuming now that $N^{2}$ is different from zero only in a sharp region $\varepsilon$ at $z=T$, one has $N^{2} \sim \delta(z-T)$. The solutions $\left(^{20}\right)$ are exponentials in $z$ and there is a dispersion relation for the internal waves:

$$
n^{2}=g k \cdot \frac{\Delta o}{\varrho^{(0)}}[\operatorname{cotgh} k T+k \varepsilon+\operatorname{cotgh} k(d-T)]^{-1}
$$

If now $l i \varepsilon \ll 1$ one has

$$
n^{2}=g l \cdot \frac{\Delta o}{\varrho^{(o)}}[\operatorname{cotgh} k T+\operatorname{cotgh}(d-T)]^{-1}
$$

and if now one assumes that $T \ll d$, which is a reasonable relation, then one has

$$
n^{2}=\frac{\Delta o}{Q^{(o)}} \frac{a k}{1+\operatorname{cotgh} l i T}
$$

It is well known that the thermocline quites the surface motions. In fact, calculating the $x$-component of the velocity $v$, one has ( $\left.{ }^{21}\right)$

$$
\begin{gathered}
u=U(z) e^{i(k x x-n t)} \\
\frac{\mathrm{d}}{\mathrm{d} z} U(z)-n k a\left(\frac{N^{\top} \mathrm{e}}{n^{2}}-1\right)
\end{gathered}
$$

where $a$ is the amplitucle of the motion of the thermocline. Then one can see how the surface motions are smoothed from the strong stratification. It is also of interest for deep oceans the case $N^{2} \sim$ const. In this case one has

$$
\begin{gathered}
W(z)=A e^{i k_{3} z}+B e^{-i k_{3} z} \\
k_{3}=k \mid \frac{N^{2}-n^{2}}{n^{2}}
\end{gathered}
$$

One can also write this relation as

$$
n= \pm N \frac{\mathbf{k}}{\mathbf{k}} \cdot v
$$

where $\gamma$ is one horizontal versor. This formula could have been intuitively obtained by the electromagnetic analogy: the coriolis force corresponds to the magnetic field. There are some other explicit potentials which give some explicit dispersion relations. They correspond usually to well-known examples of quantum mechanic's. Their utility in practical cases decreases by the usual occurence of currents, which complicate the equations. 


\section{THF SPECTREM OF TIIF INTERNAL WAVES}

At the light of the precerling consirlerations, we now describe the study of Garrett and Munk (6,7). They synthesized all known measurements about the various internal waves which can be seen at the same time. This because the various origins of internal motions give in every time and every point of the sea many internal waves. It has to be remarked also that is not very easy for the friction or other effects to destroy the internal waves ( ${ }^{(0.7)}$ so we are rather in presence of something like an equilibrium. This is in some sense the rather surprising result of these authors.

The studied measures are of two types, moored and towed. There are also correlations at various distances. There are some a-priori hypothesis i) observations of different seas and different periods are considered; ii) an horizontal hysotropy is assumed in general; iii) the internal motions are considered as random compositions of elementary internal waves; iiii) the turbulence is not consiclered, although it probably influences the problem; however the same formulation is very fruitful to describe synthetically the experimental data and to suggest new experiments.

In formulate, the various experimental data can be expressed as integrals of the various physical quantities.

This is correlaterl to the probability of having an $A$ exp $[i(k x-n t)]$ wave and is obtained from the comparison with the experiments. It results

$$
\begin{aligned}
& E(k, n)=6.310^{-5} \frac{1.5}{\left(1+\frac{k}{k^{*}}\right)^{2.5}} \frac{1}{k^{*}} \frac{2}{\pi} \frac{\Omega}{n^{2}} \frac{1}{\sqrt{1-\frac{\Omega^{2}}{n^{2}}}}= \\
& =610^{-5} \frac{1}{\left(1+\frac{k}{k^{*}}\right)^{2.5}} \frac{1}{k^{2}} \frac{\Omega}{n} \frac{1}{\sqrt{n^{2}-\Omega^{2}}}
\end{aligned}
$$

where $\Omega$ is the Coriolis parameter and

$$
k^{*} \cong 6 \pi\left(n^{2}-\Omega^{2}\right)^{1 / 2}
$$

It has to be stressed again the experimental and empiric character of these formulat the authors have improved various times their results from the 1972 version ( $\left.{ }^{7}\right)$. 


\section{THE GeNERATION OF THE INTERNAI WAVES}

We review now the main sources of internal waves. Among various origins, the air-water interaction can give three elementary mechanisms (26):

1) moving pressure field

2) a strong wind gives a stress field

3) moving inlomogeneity of buoyancy flux at the sea surface (18).

Iet us start by studying the effect of a moving pressure field on a stratified fluid. For a two-layer fluid there is the above mentioned (lispersion relation $\left({ }^{3}\right)$.

$$
n^{2}=\frac{\Delta \varrho}{\varrho_{0}} \frac{g h:}{1+\operatorname{cotgh} k T}
$$

the corresponding phase velocity for the internal waves is:

$$
\frac{n}{k}=\sqrt{\frac{g}{k} \frac{\Delta \varrho}{\varrho_{0}} \frac{1}{1+\operatorname{cotgh} k T}}
$$

Iset us now call

$$
P=P_{o} \sin (\alpha x-\sigma t)
$$

the atmosphere air moving pressure field. Now, if $\frac{n}{k}<\frac{\sigma}{\alpha}$ some transversal internal waves are generated. They are divergent and in some sense could be compared with the surface waves left by a ship (14). If at the contrary $\frac{n}{k}>\frac{\sigma}{a}$, there camnot be waves travelling in the direction of the pressure field and then could also exist some waves perpendicular to the field. One could remained also some aspects of the Cerenkov effect. This happens when a charged particle arrives on a merlium where the velocity of light is smaller than the velocity of the charged particle.

The last case $\frac{n}{k}=\frac{\sigma}{\alpha}$ represents a resonance between internal and external fields: the rate of increase of the internal wave amplitude can be computated $\left({ }^{15}\right)$. The result allows an interesting comparison with other effects $\left({ }^{3}\right)$. About the effects of the wind stress, one can say that waves of frequency larger than the initial waves are mainly clue to vertical motions which are raised by the winds $\left({ }^{2 \pi}\right)$. The rate of increase of the amplitude for the internal waves is obviously propor- 
tional to the wind stress and to the phase relocity of the waves (11). This type of knowledge appears not very satisfactory.

Then could also be a strong exchange of heat between air and water Let us suppose that the resulting variations of temperature are

$$
T \sim e^{i(k x-\sigma t)}
$$

This could give some resulting movement of the intemal layers $\left({ }^{28}\right)$. $\Lambda$ numerical comparison of the various rates of growth can show, however, that this effect is not as large as that due to the wind stress, which is practical results the most important one ${ }^{(26)}$.

A part from the air-water interactions, one can extract some other elementary sources. There could be some instability of some shear. We will not discuss it here because it could be in some sense considered as a case of turbulence. There is a non-linear exchange of energy among waves $\left({ }^{26}\right)$.

There could also be the effect of a submarine mountain on a current. This phenomenon could be similar to the "lee waves" generated on a wind by a mountain (io). Where is a mountain teller than the boundary layer, after the mountain one can see in both cases some stationary waves, called "lee waves". These could be both horizontal with an angle with the horizontal surface $\left({ }^{20}\right)$. By a theoretical point of view, one could study the inviscid stearly two dimension Fuler equation for a stream function $\psi$

$$
\Delta \psi=F(\psi)=a \psi+b
$$

This method is due to Long and it has been semplified by Yih $\left.{ }^{8}\right)$. The general function $F$ is suggested by the theory; the more practical linearization is in the common use. One could however criticize the effective solidity of these solutions by considering also in general the boundary condition $\left({ }^{1,24}\right)$.

A last aspect of remarkable interest concerns the non-linear exchange of energy among waves. This field is not only interesting by an experimental point of view but also because it appears useful (or perhaps necessary) to introduce modern tools of theoretical analysis $\left({ }^{25}\right)$.

The physical effect concerns the non-linear kinematical terms of the Euler equation, which is used to describe the elementary waves. This happens both for surface and internal waves ( $\left.{ }^{17}\right)$.

These important results are that the physical quantity related to the Lagrangian is the pressure and not, as one wauld expect, the energy. 
The starting point has been found theoretically by Phillips $\left({ }^{21}\right)$, theoretically because the measures are quite difficult and have been done only after this work. Phillips studied systems of $n$ surface waves, that are $n$ elementary solutions of the Euler equation linearizen. He studied in particular the effect of the non-linear kinematical coupling among these elementary waves. In the classical case of surface waves he shown that for $n \geqslant 4$ the system is not stable because one of the waves increases its energy at less of the others. The calculations being clear in abstract, they are in practice quite laborious. We will stress some most striking aspects of this phenomenon. The key relation is (21)

$$
\sum_{i=1}^{n} k_{i}=\sum_{i=1}^{n} w_{i}=0
$$

where the wares are

$$
e^{i\left(k_{i} x-w_{i} l\right)} \quad i=1, \ldots n
$$

If this relation is satisfeid, then the phenomenon of exchange of energy appears. It can be shown that for the case $n=2, n=3$ for surface waves the two relations cannot be satisfied at one time. This is because there could be resonance only for $n>4$. If there is at the contrary a mixing of surface and internal waves, one could also find solutions for $n=3$. By an analytical point of view, the equations are a system of $n$ nonlinear equation of erolution. It has been rerified in the following how good results the theory gives. Its predictions results $\left(^{21}\right)$ reasonably good: an interesting experimental application was found in the instability of a pure stokes ware. Its instability being before considered of experimental origin, then it turned out to be due to the intrinsic instability of the solution of Ievi-Civita et al. $\left({ }^{21,28}\right)$. It is rather curious that a longly studied solution like this results at the end non stable!

One can generalize on theoretical ground the preceding results in two ways. Either one introduces following Iuke ( $\left.{ }^{17}\right)$ and Whitham $\left({ }^{30}\right)$ a variational formulation or one studies a system of infinite gaussian waves in the quantum field formalism following Hasselmann ( $\left.{ }^{9}\right)$. In the variational formulation, use is done of a Iagrangian due to Luke (17)

$$
\mathcal{L}=\frac{\partial}{\partial t} \phi+\frac{1}{2}(\nabla \phi)^{2}+g z
$$

for the equation of motion and the boundary conditions relative to the wave motion. It is obvious that a Lagrangian allows variational calculations which are much easier. A rather surprising feature of these important results is that the physical quantity related to the Lagrangian is the pressure and not, as one would expect, the energy. 
Some application of the method to a Stoke wave shows the strength of the methorl $\left({ }^{30}\right)$. For the internal waves such technique is non complex: one has to introduce a Clebsh transformation (16)

$$
\mathbf{v}(\mathbf{x}, t)=\nabla \phi(\mathbf{x}, t)+\lambda(\mathbf{x}, t) \nabla \mu(\mathbf{x}, t)
$$

where the potentials are now $\phi, \lambda$ and $\mu$. This gives a major complexity of the treatment.

Some general problems can be solver by using in general these improvements of the theoretical technique. It appears interesting the use of the two-time methor. This consists in remarking that the phenomenon has two important scale length. One concerns the wave lengths. The other concerns the time of variation of the ampliturle frequency and wave length for a wave train. With a rather complex mathematical treatment these two times are recoupled and the slower one can be analyzed. The resulting equations are series expansions. The first term seems reasonable but it is not very clear the general validity of these results. This happens in particular for the very interesting discussion of Whitham for the difference between phase and group velocities. Interester to an other physical situation, a realistic case of an infinity of waves with Gaussian ristribution, Hasselmann has done a perturbative calculation till the 5th order. He obtains a relation that can be generalized to an equation. This results a nonlinear integral equation in the wave number $k$ and rifferential in the time $t$. The calculations being rather heary, the generalizations have not been very numerous $\left({ }^{9}\right)$.

For surface wave IIasselmann not only finds the above rlescribed equation, but he has also been able to fix some conserved quantities

$$
\begin{aligned}
& \int E(k) d \mathrm{k} \text { the energy } \\
& \int \mathbf{k} E(k) \text { llk the momentum } \\
& \int \frac{E(k)}{w(i)} \text { dk which physical interpretation is more rifficult. }
\end{aligned}
$$

In more general terms Hasselmam has generalized his results to every kind of waves, by using the very natural formalism of the quantum field theory. In this context it's natural to call $\frac{W}{w} \sim n$ like a number of excitations or waves. Then the last relation conld have the meaning of a conservation of number of waves. The relation of the work of Hasselmann $\left({ }^{9}\right)$ with the Luke $\left({ }^{17}\right)$ formalism is not very easy. 


\section{REFERENGES}

(1) BARCIION V., 1971. - A simple model of the thermocline in a bounded Ocean. "Journ. of Phys. Ocamogr.", 1, pp. 7.11 .

$\left({ }^{2}\right)$ Canor 1"., 1959. - Termologin del lago di Bracciano: omde interne. "Annali di Gicofis.", XII, 1, pp. 1-19.

${ }^{3}$ ) Catol P', 1963. - Interazioni tra almosfera e idrosfera. "Annali di Geofis.", XVI. 1, 137-178.

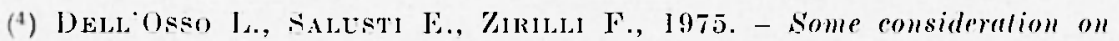
the usual derivation of the I'ocliels (or Helmhotz) equation sleadly tino dimensional Rnid dynamics. "Annali di Cieofisiea", XXVIII, 1, pp.

${ }^{5}$ ) Dexuaxi K. L., 1973. - A time-dependent molel of the upper Ocent. "Journ. of Phys. Oceanogr.", 3, pp. 173-184.

$\left({ }^{6}\right)$ GarketT C. J. R., MuNi W. II., 1972. - Space-lime scales of internal wares. "G(cophlys. Fluid DỴn.", 2, pl). 225-264.

(7) Garketт C. J. R., IU:k W. II., 1975. - Space-lime scales of imtermal wares: a progress report. "Joum. of (icophys. Rescarch", 80, pp. 291-297.

$\left.{ }^{8}\right)$ (iUTMAx L. X., 1972. - Introduction to the non linear theory of mesoseale meteorologieal processes. "Istael Program for scientifie 'Translation".

$\left({ }^{9}\right)$ Hassemasx K., 1962. - On the non-linear energ! transfer in "gravily ware spectrum. "Journ. of Fluid Mech.", 12. ple. 481-500.

$\left.{ }^{10}\right)$ IIoLrox J., 1972. - An introduction to dynamie melheorology. "Academie Press", Now York, landon.

(11) Hudnac A. A., 1961. -- ship wares in a stralified Ocean. "Jomm. Fluid Mcch.", 11, p). 229-243.

(12) Krauss E. B., Tunser J. S., 1967. - I one dimensional model of the seasonal thermocline I: a laboratory erperiment and its interprelation. "Tollus", XIX, pp. ss-97.

(13) Krauss E. B., Turser J. S., 1967. - A ome dimensional model of the seasonal thermocline $I I$ : the general theory and its consequences. "Tal. lus", XIX, pp. 98-105.

(14) Kratss E. B., 1972. - -1/mosphere-Ocean Interaetion. Clandondon P'ress, Oxford.

(15) Krauss W., 1972. - On the response of a stratified Ocean to wind and air pressure. "Deut. Hridrogr. Z.". 25. pp. 241-250.

${ }^{(16)}$ Janis H., 1932. - "Ilydrodynamies". Cambridge, lniv. Press, Xow louk.

${ }^{(17)}$ LUk: J. 6., 1967. - I rariational principle for a fluid with a free surface. "Journ. Fluid Ifech.", 27. pp. 395-397.

(18) IAGAARD L., 1973. - On the generation of intermal gravity wares b!y " fluctuating buoyaney fher at the sea surjace. "Geophys. Fluid Dyn.", 5(2), 11). 101-111: 
(19) Nardose M., 1976. - Preprint no 73. Istituto di Fisica "G. Mareoni", Universiti degrli studi - Roma.

(20) Neumaxx G., Prersox W. J., 1966. - Principles of physical Oceanography. "Prentice-Hall", Now Jersey.

(21) Phildips O. M., 1966. - The dynamies of the upper Ocean. "Lniv. Press", Cambrilge.

(22) l'HuLLips 0. M., 1971. - On spectra measured in a ondulating layered medium. "Journ. of Physic. Oceanogr.", 1, pp. $1-16$.

(23) Robissox A., Sтомие H., 1959. - The oceanic thermocline and the associated thermocline circulation. "Tellus", XI, pp. 295-308.

(24) Salusti E., Deld Osso Y., 1973, - On some rigorous effects in non-linear steady fuid-dynamies. Nota interna, Ist. di Fisica "G. Mareoni", Università di Roma.

(25) Srhiger R. L., Whitha G. B., 1968. - Tariational prineiples in continum mechanics. "Proc. Roy. Soc.", A. 305, pp. 1-25.

(26) THonpe S. A., 1975. - The excitation, dissipation, and interaction of internal waves in the deep Ocean. "Journ. of Geophys. Research", 80 , pp. 328-338.

(27) ToмcZak M. Jr., 1966. - Winderzengle interne Wellen, insbesondere im Periodenbereich oberhalb der Tragheitsperiode. "Dent. Hydrog. Z.", 19, pp. 1-21.

(29) Turxen .J. S., 1973. - Buoyancy effects in Fluids. "Univ. Press", Camhridge.

(29) WELAxisr P', 1959. - An advective model of the ocean thermocline. "Tellus", XI, pl. 309-318.

(30) Wniman G. B., 1974. - Linear and non-linear waves. "J. Wiley \& Sons", New York.

${ }^{(31)}$ Woons J. D., 1968. - Wave-induced shear instability in the summer thermoctine. "Journ. of Fluid Mechanies", 32, pl. 791-800. 